\title{
Acoustic-emission method for controlling the defect-formation process in light-emitting structures
}

\author{
O.V. Lyashenko*, A.I. Vlasenko, V.P. Veleschuk, M.P. Kisseluk \\ V. Lashkaryov Institute of Semiconductor Physics, NAS of Ukraine \\ 41, prospect Nauky 03028 Kyiv, Ukraine \\ Phone:+38(044)-5258437; e-mail:vvvit@ukr.net \\ *Taras Shevchenko Kyiv National University, \\ 2, Academician Glushkov Ave., 03022 Kyiv, Ukraine; \\ E-mail:lyashenk@mail.univ.kiev.ua
}

\begin{abstract}
Complex researches of light-emitting structures based on $\mathrm{A}_{3} \mathrm{~B}_{5}$ compounds have been carried out. It has been shown that at current loading exceeding the acousticemission threshold, there arises a change in the electroluminescence intensity, fluctuation of current and light. It has been ascertained that natural ageing leads to a general improvement of some practically important parameters of light-emitting structures, in particular to increase of maximal admissible currents and reliability.
\end{abstract}

Keywords: acoustic emission, light-emitting structure, electroluminescence.

Manuscript received 18.12.09; accepted for publication 08.07.10; published online 30.09.10.

\section{Introduction}

Devices based on the light-emitting diode structures are intensively used in information displays as elements of displays and illumination. Simultaneously with reduction of price and increase of efficiency of these structures, there arise actual problems of using these structures in devices with short-term service life, but as much as possible using already available opportunities of lightemitting structures - for example "disposable" devices in medical technologies.

One of the most promising materials to create super-bright light-emitting diodes (LED) of blue, green and white luminescence is gallium nitride based solid solution. To increase the light flux and efficiency, except for improvement of materials quality, improvement of technology and optimization of structure formation and its ohmic contacts, application of periodic multiple quantum wells, proved is increase in the area of a radiating structure.

Besides, to enhance the electroluminescence (EL) of light-emitting structures and, accordingly, lightemitting diodes in practice, it is possible by increasing the density of direct current through heterojunction $J$. However, then the following problems become more essential.
1. Non-uniformities in $J$ distribution over an active area of the structure, including any section of the current tube (in particular, the "current crowding" effect [1]).

2. Local overheating in the active area that is already overheated by tens-hundreds degrees as compared to other areas of this heterostructure.

3. And, on first glance, the most important - some reduction in the service life of devices, first of all because of thermal and electric activization of diffusion processes inherent to impurities and dot defects in the active area, and metal to semiconductor in the vicinity of contacts, as well as processes providing formation of defects (both point and structural) in the active and contact areas for high $J$ values.

The above mentioned processes lead to degradation, in particular, reduction of the injection coefficient, slowing down the diffusion length of carriers and their lifetime in $\mathrm{p}-\mathrm{n}$ transition and, accordingly, irreversible degradation of a light flux caused by increase of radiationless recombination, as well as decrease in the efficiency of electric power-to-radiation transformation.

It is known that in the active area of LED the gradient of temperature reaches $10^{4} \mathrm{deg} / \mathrm{cm}$ [1], and even 
$10^{5} \mathrm{deg} / \mathrm{cm}$, which taking into account the difference of thermal expansion coefficients $\alpha_{i}$ of separate layers and their lattice constants $a_{i}$, thermal resistance of the structure $R_{t c}$ and case $R_{t k}$ results in creation of local plastic thermo-mechanical strains in $p-n$ transition when current flows. But a sharp drop (fast relaxation) in these induced thermo-mechanical strains leads to occurrence of spontaneous chaotic radiation of acoustic waves with a noise character - to acoustic emission (AE) of these materials caused by local dynamic reorganization of the structure, i.e. defect-formation [2, 3].

Since the nondestructive (and, in addition, not influencing on the chosen operating modes of semiconductor devices and parallel methods of their research) acoustic-emission method providing the control of light-emitting structures allows to make the control of velocity of defect-formation processes (and also, together with other methods, degradation of structures [4]) in a real time mode, therefore, to develop an express version of the method needs initial, basic researches.

Thereof, the goal of this work was to analyze dynamics of local defect-formation processes in lightemitting structures induced by direct constant current, which result in simultaneous changes in EL intensity distribution over radiation surface, AE occurrence, oscillation of integrated EL intensity and current fluctuations, as well as to general degradation of electrophysical parameters of heterostructures.

\section{Results and discussion}

Shown in Fig. 1 are EL spectra of industrial InGaN/GaN structures of blue (a) and green (b) radiation. The EL intensity $I$ increases up to saturation when the current $J$ grows within the range $90-100 \mathrm{~mA}$. Further increase in $J$ leads to some reduction of $I$, in what follows, one observes accelerated degradation caused by active defect-formation, which is accompanied by more intensive AE.

Since, despite the decrease in $I$, the researched structures kept operation ability, we carried out researches of dependences of permanent resistance $R_{s}$ and light flux $L$ values on the operation time $t$ at $J=160 \mathrm{~mA}$ (Fig. 2). It is seen that there observed are simultaneous monotonous increase in $R_{s}$ and insignificant decrease in $L$ values, but in the maximum of $R_{s}(t)$ one can see synchronous sharp drops in $R_{s}$ and $L$. In this moment, there takes place some increase in the AE intensity, which simultaneously is accompanied by fluctuations $\Delta L$ and $\Delta J$ (Fig. 3).

It is necessary to note that in these structures above $110 \mathrm{~mA}$ simultaneously with $\mathrm{AE}$ signals and $\Delta I\left(S_{i}\right)$ registered also are integrated (over all surface of the structure) fluctuations of $\Delta I(S)$ and current fluctuations.

Usually, the following correlation between AE, $\Delta L$ and $\Delta J$ is observed: actually every group of AE signals is corresponded with a change of excess current noise and fluctuations of the integrated (both over the surface and radiation spectrum) EL intensity $\Delta I(S)$ (Fig. 3). The given signals are processed by the program for elimination of the own noise of equipment for registration of changes - fluctuations.

Earlier [2], it was ascertained by us for the first time that operational dynamics of AE sources in lightemitting structures based on the GaAsP, GaP and GaAlAs compounds undergoes significant influence of processes of natural ageing that for time $6 \cdot 10^{8} \mathrm{~s}(20$ years) considerably reduce total AE intensity, raise 10 to 20 -fold the maximal admissible current densities. In particular, it concerns the density of destruction currents and AE threshold. It is indicative of the defect subsystem transition in the structure to other metastable state with a higher activation energy $E_{a}$. In fact, there takes place an increase in local strength for operating thermo-strains created by local current heating, due to significant increase of $E_{a}$ in newly formed complexes of defects. At natural ageing, there is also a significant growth of adhesive durability of metal contacts to the semiconductor [4], which is indirectly confirmed by the fact of significant increase in destruction currents of structures with time in our investigations $[2,3]$.
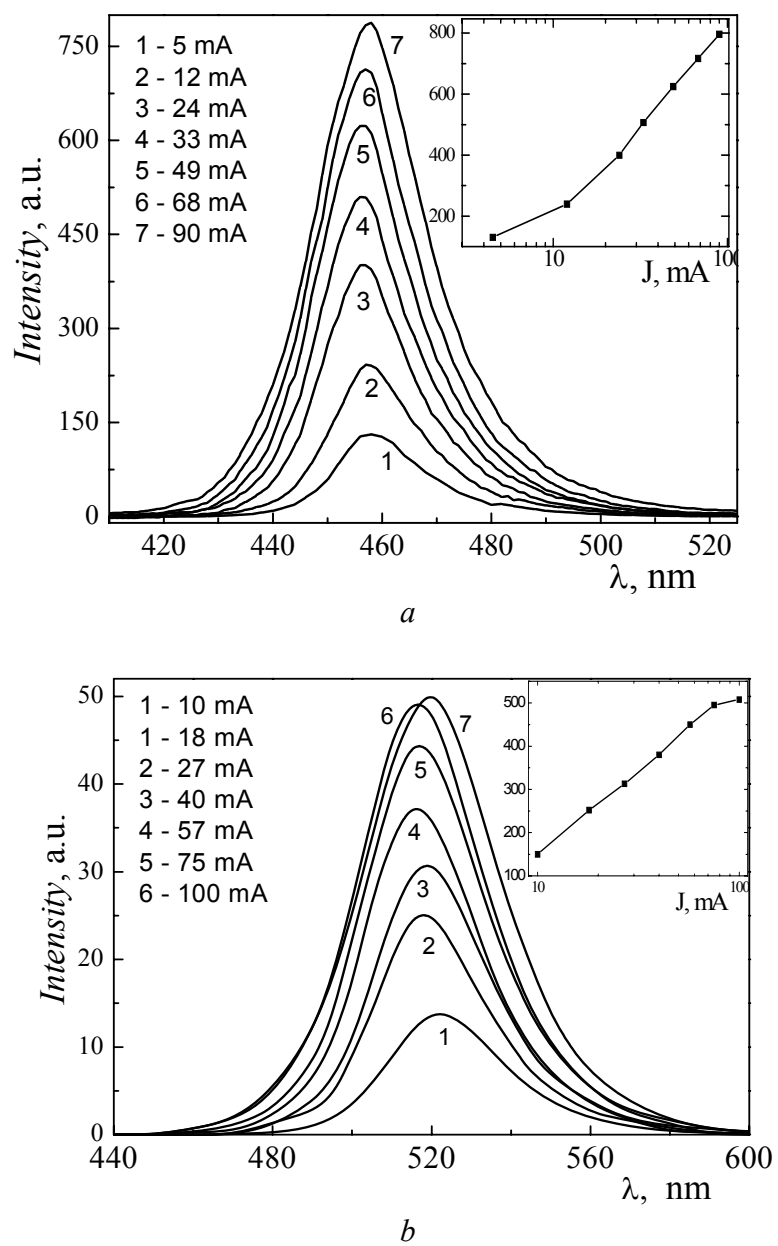

Fig. 1. EL spectra $I(\lambda)$ of blue $(a)$ and green $(b) \operatorname{InGaN} / \mathrm{GaN}$ light-emitting diodes. In inserts $-L(J)$. 


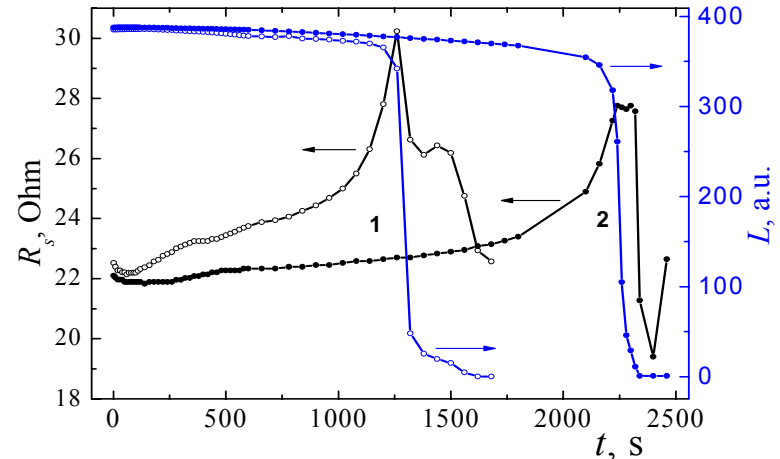

Fig. 2. Dependence of the permanent resistance $R_{c}$ and light flux $L$ on $t$ at $J=160 \mathrm{~mA}$ for two LEDs $(1,2)$.
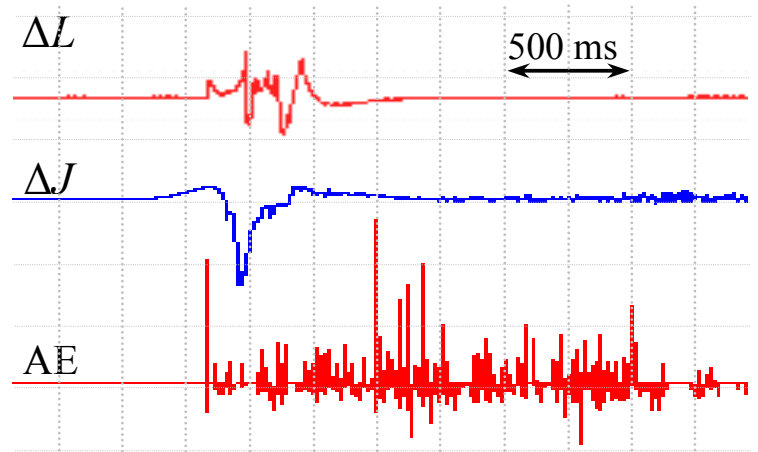

Fig. 3. AE, fluctuations of the light flux $\Delta L$ and current $\Delta J$ of InGaN/GaN structure at $J=160 \mathrm{~mA}$ in the moment $t=1254 \mathrm{~s}$ in Fig. 2, curve 1.

\section{Research of high power white and blue light-emitting diodes}

Up to date, high power blue and white LED (with the active area $1 \mathrm{~mm}^{2}$ ) intensively comprises the range of light engineering and especially lamps for general white lightning. As said above, to enhance a light flux of lightemitting diodes in practice, it is possible by using a higher density of current through heterojunction. But, at the same time, the problems mentioned in introduction remain.

Shown in Fig. 4 is the dependence of EL intensity for peaks of radiation bands in the high power $\mathrm{InGaN} / \mathrm{GaN}$ structure $(467 \mathrm{~nm})$ and luminophore covering it $(583 \mathrm{~nm})$. Represented in the inset is the LED spectrum at $350 \mathrm{~mA}$. The LED with proper radiator was placed on an additional metal radiator with the sizes $3 \times 3 \times 0.4 \mathrm{~cm}$ fixed with a metal holder to a table. The temperature $(T)$ of metal contacts for LED at $2 \mathrm{~A}$ did not exceed $230^{\circ} \mathrm{C}$. In this case, a maximum is seen at $1400 \mathrm{~mA}$, at this current the LED continuously operated for more than 2 hours without degradation. The temperature of metal contacts was $110^{\circ} \mathrm{C}$. The intensity of the blue band of InGaN/GaN structure after $1400 \mathrm{~mA}$ falls more slowly, than that for white luminophore (Fig. 4). It was clearly seen that the color temperature of light changed from "warm" to "cold".
Fig. 5 shows the current-volt characteristic (CVC) of blue LED without additional radiator. This CVC has a part of simultaneous reduction of current and growth of direct voltage, which corresponds to irreversible growth of differential resistance with time due to formation of defects. This formation is observed for induced by current (owing to non-uniform local heating) relaxation of non-uniform thermo-mechanical strains at constant external loading. Shown in the same figure is the temperature of metal contacts: it is seen that the temperature practically has the same dependence as a current, which was observed for all LED of this type. In the point marked $\nabla$ (Fig. 5) where the CVC dependence has peculiarity and further, $T$ begins to increase at $U_{\text {external }}=$ const, which is indicative of the growing resistance due to defect-formation processes in the structure, like to that observed in [3].

At the same time, on CVCs of four white LEDs of two different manufacturers the given part of drop was absent down to $2 \mathrm{~A}$ ( $T$ was approximately $240^{\circ} \mathrm{C}$, i.e., the melting point of $\mathrm{Pb}$-Sn solder), while 4 LEDs have failed due to melting of one of contacts (whiskers). When the contacts had been broken, AE was registered.

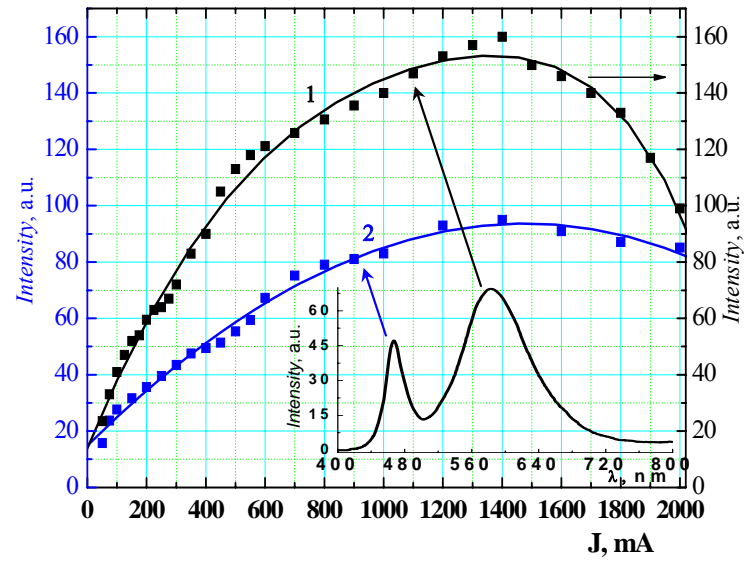

Fig. 4. Dependence of the EL intensity peaks on current for white LED and its spectrum. $1-\lambda_{\text {peak }}=583 \mathrm{~nm}, 2-\lambda_{\text {peak }}=$ $467 \mathrm{~nm}$.

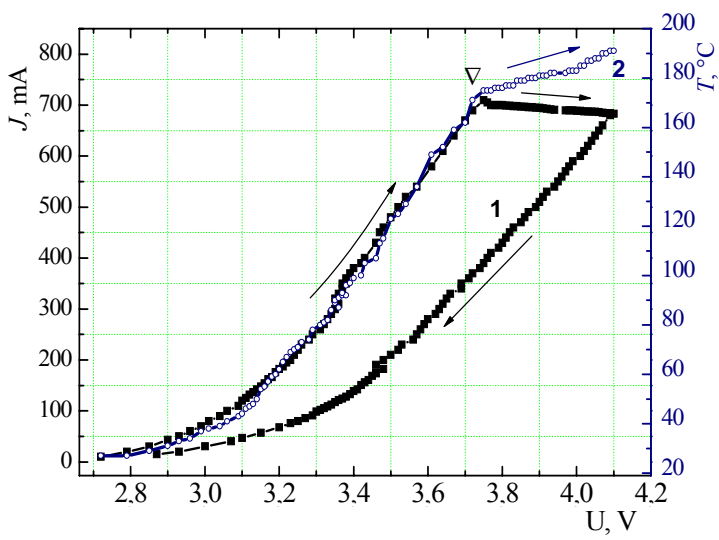

Fig. 5. CVC (1) and temperature of contacts (2) for a blue LED. 


\section{Conclusions}

Thus, it has been shown that application of the acousticemission method allows to predict formation of defects in light-emitting structures when current passes at an early stage of the defect-formation process and, accordingly, to predict their reliability if operating in extreme modes. AE occurrence testifies to activation from one- up to three-dimensional defects in these lightemitting structures.

From the complex researches of light-emitting structures, in particular InGaN/GaN, it follows that at current densities which exceed the threshold of $\mathrm{AE}$ occurrence, simultaneously observed are: operation of $\mathrm{AE}$ sources, irreversible change in the EL intensity and fluctuations of the light flux and current. It is indicative of the common mechanism of their origin - processes of occurrence (or changes in the state) of extended defects.
References

1. A.V. Zinovchuk, O.Yu. Malyutenko, V.K. Malyutenko, A.D. Podoltsev, A.A. Vilisov, The effect of current crowding on the heat and light pattern in high-power AlGaAs light emitting diodes // J. Appl. Phys. 104, 033115 (2008).

2. V.P. Veleshchuk and O.V. Lyashenko, Acoustic emission of light-emitting structures on the $\mathrm{A}^{3} \mathrm{~B}^{5}$ base determined by direct current // Ukrainsky fiz. zhurnal 48(9), p. 981-985 (2003) (in Ukrainian).

3. A.I. Vlasenko, O.V. Lyashenko, P.F. Oleksenko and V.P. Veleschuk, Fluctuations of current, electroluminescence and acoustic emission in lightemitting $\mathrm{A}^{3} \mathrm{~B}^{5}$ heterostructures // Semiconductor Physics, Quantum Electronics \& Optoelectronics 11(3), p. 230-235 (2008).

4. E.F. Venger, R.V. Konakova, G.S. Korotchenkov, V.V. Milenin, E.V. Russu, I.V. Prokopenko, Interphase Interactions and Degradation Mechanisms in Metal-InP and Metal-GaAs Structures. Kyiv, "KTNK”, 1999, p. 260 (in Russian). 